\title{
COMPARAÇÃO ANATÔMICA DA ENERVAÇÃO DA ARTICULAÇÃO COXO-FEMORAL EM HUMANOS E CÃES
}

\section{Anatomic Comparison of the innervation of hip joint in human and dogs}

SCHMAEDECKE, A.. ${ }^{1}$; FERRIGNO, C.R.A. ${ }^{2}$

${ }^{1}$ Departamento de Medicina Veterinária - UFPR
${ }^{2}$ Departamento de Clínica Cirúrgica - FMVZ, USP

Endereço para correspondência: Alexandre Schmaedecke - alexandre_vet@hotmail.com

\section{RESUMO}

Foi comparada a disposição das fibras nervosas responsáveis pela cadeia sensitiva de cães e seres humanos, uma vez que técnicas cirúrgicas vêm sendo desenvolvidas para os pacientes veterinários, baseadas em similares utilizadas no controle da dor de quadros de artrose de seres humanos. Destaca-se nesta revisão que em seres humanos a enervação sensitiva da região acetabular é composta por ramos oriundos dos nervos glúteo superior, isquiático, femoral e obturador, enquanto em cães são responsáveis os nervos glúteo cranial, isquiático e femoral, estando o nervo obturador não relacionado a esta função na maioria dos animais, podendo ser assim considerado variação anatômica. A enervação tanto em seres humanos como em animais apresenta simetria bilateral e, além disso, que a diferença entre o apoio bipedal humano e quadrupedal canino gera forças biomecânicas em pontos distintos, promovendo diferente concentração de fibras nervosas em relação às duas espécies. Enquanto a primeira apresenta maior densidade de fibras nervosas na região anteromedial, de responsabilidade do nervo obturador, a segunda apresenta maior densidade na região crânio-lateral e dorsal, de responsabilidade dos nervos glúteo cranial e isquiático.

Palavras-chave: acetábulo, nervos periféricos, cães, humanos, anatomia.

\section{ABSTRACT}

The arrangement of the nerve fibers responsible by the sensitive route of dogs and human beings were compared, since surgical techniques that have been developed for animal patients are based in similar ones used for pain control in arthrosis cases in human beings. In this review it was outstanding that in human beings the sensitive innervation of the acetabular area is composed of branches from the superior gluteal, ischiatic, femoral and obturator nerves, while in dogs the responsible nerves are the cranial gluteal, ischiatic and femoral nerves. The obturator nerve is not related to this function in most animals, hence it can be considered an anatomic variation. The innervation, both in human beings and animals, presents bilateral symmetry and, apart from that, the difference between the human bipedal support and the canine quadrupedal support generates biomechanical forces in distinct points, leading to different nerve fiber concentrations between the two species. While the first presents a bigger nerve fiber density in the anteromedial area, for which the obturator nerve is responsible, the second presents a bigger density in the craniolateral and dorsal areas, for which the cranial gluteal and the ischiatic nerves are responsible.

Key words: acetabulum, peripheral nerves, dogs, humans, anatomy. 


\section{INTRODUÇÃO}

O estudo da enervação da articulação coxo-femoral em cães se tornou essencialmente importante após comprovações iniciais de que a simples remoção do periósteo acetabular promove a remissão dos quadros de dor de pacientes com displasia coxo-femoral, com retorno à atividade funcional normal do paciente e conseqüente melhora da qualidade de vida. A diferença entre o apoio bipedal e plantígrado humano para o quadrupedal digitígrado dos cães gera ângulos articulares diferentes entre estas espécies, bem como permite a suposição da diferença de enervação das articulações de forma geral e da região coxo-femoral em específico, haja vista que diferentes forças biomecânicas atuarão nesta articulação.

A presente revisão de literatura teve como objetivo comparar a enervação das articulações coxo-femorais de cães e seres humanos, apresentando as diferenças significativas da disposição nervosa em relação às espécies citadas.

\section{A ENERVAÇÃO DA ARTICULAÇÃO COXO-FEMORAL HUMANA}

Os estudos de Gardner (1948) mostraram que a maior concentração de terminações nervosas está localizada onde as maiores forças de compressão estão atuando. Aquele autor descreve ramos articulares do nervo femoral, inserindo-se pelo músculo pectíneo, em direção ao reto femoral e vasto lateral. Em suas dissecções, em fetos humanos, Gardner (1948) cita que o ramo do músculo pectíneo acompanha a artéria circunflexa lateral, seguindo para a região anteromedial da cápsula articular. Após penetração na camada fibrosa da cápsula, ramifica-se, acompanhando vasos sangüíneos para o tecido sinovial e regiões circunvizinhas ao fêmur. Descreve ainda que este ramo direcionado do pectíneo se origina do nervo femoral em 3 dissecções, enquanto em outra se originou do nervo obturador acessório. A existência deste ramo articular foi confirmada segundo 0 autor, em dissecções de cadáveres adultos.

Os ramos do nervo femoral que caminham para o quadríceps femoral, segundo Gardner (1948) e Kenny et al. (1999) percorrem invariavelmente o músculo reto femoral, mas freqüentemente dividem-se em ramos para o músculo vasto lateral. O feixe nervoso do músculo reto lateral corre lateralmente e cruza o músculo iliopsoas adjacente ao ramo da artéria circunflexa lateral. No tecido adiposo posterior ao músculo reto femoral, este ramo divide-se em dois, muito finos, que penetram nas regiões medial e lateral do ligamento iliofemoral, próximas à sua inserção femoral.

Os mesmos autores descreve ramos articulares provenientes de um tronco maior do nervo obturador, vindos diretamente do forame obturador, tendo ocasionalmente um ramo adicional proveniente de divisão anterior. Estes ramos se dividem em feixes mais finos que penetram o ligamento pubocapsular. Tais relatos corroboram os estudos de Putz e Schrank (1998).

Já em relação ao nervo glúteo superior, Gardner (1948) descreve um ramo chegando através do músculo glúteo mínimo ou através do músculo tensor da fáscia lata. Descreve este como um pequeno ramo que acompanha vasos sangüíneos para a região superolateral da cápsula.

Tais estudos se tornaram mais importantes e discutidos após a evolução da ortopedia e neurologia clínicas e cirúrgicas, uma vez que tratamentos são propostos baseados em redução da atividade neural, principalmente em relação à enervação sensitiva articular, no controle de doenças crônicas como artroses ou controle de cirurgias protéticas.

Kawagushi et al. (2001) provocaram em 14 pacientes com dor crônica de 
quadril, não indicativa de intervenção cirúrgica, neurectomia de ramos sensitivos dos nervos obturador e femoral, através de radiofreqüência percutânea, citando a responsabilidade destes ramos em relação aos quadros de dor apresentados. Como resultados, apontaram a melhora clinica de $86 \%$ dos casos tratados, com diminuição de, pelo menos, $50 \%$ do quadro de dor inicial.

Pelo fato de muitas vezes o bloqueio do nervo obturador isoladamente não resolver os quadros dolorosos destes pacientes, conforme descrito por Akatov e Dreval (1997), Birnbaum et al. (1997) realizaram estudo anatômico da enervação acetabular, descrevendo uma separação entre a enervação sensorial das porções anterior e posterior da cápsula articular. Enquanto a enervação da região anteromedial foi descrita como determinada por ramos dos nervos obturador e femoral, a parte posterior, segundo os autores, está ligada ao nervo isquiático, associado a ramos articulares do nervo para o músculo quadrado femoral. Além destes, descrevem a enervação da região posterolateral por parte do nervo glúteo superior. Deste modo, concluem que o bloqueio apenas do nervo obturador seria insuficiente para a eliminação dos quadros dolorosos, sendo necessário o bloqueio dos nervos femoral, isquiático e glúteo superior.

Estudo embriológico desenvolvido por Golub e Bronovitskaia (1981) demonstra enervação da cápsula articular do acetábulo, vista em fetos a partir de 18 $\mathrm{mm}$ de comprimento parietal-coccigeal, proveniente de ramos do nervo obturador $\mathrm{e}$ obturador acessório em seu fragmento anterior, ramos do nervo glúteo superior em seu fragmento superior e, em seu fragmento posterior, ramos do nervo isquiático. Concluem que, dado o tempo de origem destes ramos nervosos, os mesmos devem ser considerados os principais responsáveis pela enervação capsular.

\section{A ENERVAÇÃO DA ARTICULAÇÃO COXO-FEMORAL EM CÃES}

O estudo da enervação da articulação coxo-femoral em cães tem se tornado de fundamental importância, principalmente após a descrição de novas técnicas cirúrgicas que permitem a neurotomia sensitiva da região acetabular, devolvendo ao animal a amplitude de seus movimentos. No entanto, os resultados obtidos ainda são controversos (Kinzel et al., 1998).

Staszyk e Gasse (2002) propuseram modelo baseado em enervação com as fibras nervosas penetrando por áreas distintas da cápsula articular, além de fibras nervosas "indiretas" que penetram continuamente da musculatura pelo periósteo e cápsula, o que denominaram de enervação múltipla. Perceberam ainda que, ao contrário das articulações de cotovelo e ombro, onde as enervações são assimétricas, a articulação coxo-femoral possui uma enervação bilateralmente simétrica e individualmente variável.

Kinzel et al. (1998) demonstraram em seu estudo em 16 cães, a enervação partindo de ramos articulares do nervo glúteo cranial em sua porção crânio-lateral e, em sua porção caudo-lateral, ramos oriundos dos nervos isquiático e femoral, não podendo indicar enervação do nervo obturador em nenhum cão. Schmaedecke et al. (2003) apresentaram resultados semelhantes em dissecção de 15 animais, em contraposição aos dados de Staszyk e Gasse (2002), que relatam a presença de fibras nervosas provenientes do nervo obturador em cápsula articular de cães, tendo descrito tal fato baseado nos achados de um dos animais de seu estudo. Sobre o aspecto microscópico, Schmaedecke (2004) quantificou as fibras nervosas presentes no periósteo acetabular de cães normais, tendo demonstrado como resultados a diferença estatisticamente significativa entre o número de fibras sensitivas das regiões crânio-lateral e 
dorsal do acetábulo em relação à região ventral, além de demonstrar uma penetração das fibras nervosas independentemente do feixe vascular, embora todos os feixes maiores estejam concentrados adjacentes à vascularização. Utilizando método de impregnação por prata e auxílio de software específico, identificou a densidade de fibras nervosas periosteais, com média de aproximadamente 75 fibras $/ \mathrm{mm} 2$ na região dorso-lateral, 73 fibras $/ \mathrm{mm} 2$ na região dorsal e 58 fibras $/ \mathrm{mm} 2$ na região ventral, sendo este último, valor estatisticamente inferior aos demais citados. Demonstrou ainda não haver diferença significativa entre os dois antímeros, bem como uma moderada correlação entre o número de fibras nervosas e o peso do animal.

\section{IMPLICAÇÕES INTERESPECÍFICAS DAS DIFERENÇAS ANATÔMICAS}

Dada a diferença entre o número de artigos publicados por pesquisadores da área médica em relação à medicina veterinária, torna-se evidente a importância da ciência de base como alicerce de desenvolvimento das áreas clínicas e cirúrgicas, uma vez que a aplicação de técnicas mais avançadas utilizadas pelos especialistas em pacientes humanos é notória. Esta afirmação é ainda mais comprovada quando nos deparamos com técnicas utilizadas na medicina veterinária quase que invariavelmente sendo desenvolvidas através de adaptações da medicina humana.

O modelo de enervação da região acetabular em cães pode ser definido, de acordo com os trabalhos de Kinzel et al. (1998), Staszyk e Gasse (2002) e Schmaedecke et al. (2003) como proveniente de ramos articulares dos nervos glúteo cranial, responsável pela enervação da região crânio-dorsal, isquiático na região dorsal e femoral na região medial.
Variações anatômicas desta descrição podem estar relacionadas à enervação proveniente de ramos do nervo obturador, descrita por Staszyk e Gasse (2002), embora vista em apenas uma de suas dissecções, e contrariando o proposto por Kinzel et al. (1998) e Schmaedecke et al. (2003).

Já o modelo humano, baseado em uma gama maior de pesquisas, pode ser definido por ramos articulares provenientes dos nervos glúteo superior, isquiático, femoral e obturador. O primeiro, penetrando através do músculo glúteo mínimo ou músculo tensor da fáscia lata (Gardner, 1948; Siebenrock et al., 2000), sendo responsável pela enervação da região posterolateral da articulação (Siebenrock et al., 2000). O segundo, em íntima relação com o acetábulo (Baumgaertner, 2000), torna-se responsável pela enervação da região posterior, juntamente com ramos para o músculo quadrado femoral (Birnbaum et al., 1997; Putz e Schrank, 1998). O nervo femoral emite ramos articulares através dos músculos pectíneo, reto femoral e vasto lateral (Gardner, 1948), embora estudo embriológico realizado por Golub e Bronovistskaia (1981) não cite o mesmo como um dos principais responsáveis pela enervação capsular.

O nervo obturador apresenta significativa importância na enervação da articulação coxo-femoral humana, haja vista que estudos comprovam que a neurectomia seletiva de suas fibras sensitivas melhoram significativamente a qualidade de vida em pacientes portadores de artrose não indicativa de cirurgia (Akatov e Dreval, 1997; Kawaguchi et al., 2001). Este vem enervar a cápsula articular através de ramos que penetram através do complexo labro acetabular, pelo ligamento pubocapsular.

Variações anatômicas desta descrição podem estar relacionadas à enervação capsular por parte dos nervos 
obturador acessório e ramos do nervo glúteo inferior (Gardner, 1948).

Comparativamente pode-se demonstrar a diferença de densidade de fibras nervosas na cápsula articular de humanos e cães em virtude das forças biomecânicas divergentes que atuam nos acetábulos dos objetos deste estudo. Enquanto em cães, que assumem a posição quadrupedal e digitígrada, observa-se maior densidade de fibras provenientes dos ramos dos nervos glúteo cranial e isquiático, conforme disposto por Kinzel et al. (2002) e Schmaedecke (2004), nos seres humanos, cuja postura se baseia em apoio bipedal e plantígrado, verifica-se a maior densidade das fibras provenientes do nervo obturador, conforme disposto por Gardner (1948). Tal fato é comprovado clinicamente pelos resultados dos procedimentos cirúrgicos de Kinzel et al. (2002) e Ferrigno et al. (2004) com denervação seletiva das fibras sensitivas basicamente emitidas dos nervos glúteo cranial, isquiático e femoral em cães, enquanto em seres humanos, os estudos de Kawaushi et al., (2001) demonstram a melhora clínica de pacientes tratados através de neurectomia dos nervos femoral e obturador.

À análise do nervo glúteo superior, denominado glúteo cranial em cães, é notória a disparidade entre o número de fibras que são emitidas e sua importância na enervação articular. Enquanto nos primatas, os estudos de Kenny et al. (1999) indicam ausência de correlação significativa entre lesões do nervo glúteo cranial e problemas clínicos apresentados por pacientes, as publicações de Kinzel et al. (2002) e Schmaedecke (2004) demonstram a efetiva importância deste feixe nervoso em relação ao acetábulo dos canídeos.

Em relação ao nervo isquiático, é evidente sua importância em ambos os objetos desta revisão, como pode ser comprovado pelos estudos de Baumgaertner (1999), que indica a incidência de até $18 \%$ de acometimento do nervo em questão, em fraturas acetabulares de pacientes humanos. Embora os dados veterinários não apresentem incidência específica, Jacobson e Schrader (1987) apontam a lesão do nervo isquiático como das principais conseqüências dos processos de fratura simples ou associada a deslocamento acetabular em cães.

Conforme estudo publicado por Staszyk e Gasse (2002), e confirmado por Schmaedecke (2004), ocorre uma simetria entre os dois antímeros quanto a sua enervação, fato este que pode ser extrapolado às pesquisas em seres humanos, pois nenhum dos autores refere assimetria antimérica vista em suas dissecções.

É consenso também que os feixes nervosos maiores estejam adjacentes à vascularização local, penetrando na cápsula articular através do periósteo (Gardner, 1948; Mach et al., 2002; Schmaedecke et al., 2003). A diferença encontra-se no número de fibras denominadas por Staszyk e Gasse (2002) como indiretas, oriundas de feixes musculares, que, em seres humanos é notoriamente maior que em cães. Deste modo, como o número de fibras diretas no periósteo dos cães é maior, explica-se o sucesso da cirurgia de denervação em pacientes veterinários, como relatam os números de Kinzel et al. (2002), com 90\% de bons resultados, e Ferrigno et al. (2004), com $95 \%$ de bons resultados, e a maior dificuldade em se obter números de tamanha expressão com procedimentos similares em pacientes humanos, como pode-se notar nos resultados de Kawagushi et al. (2001), que apresenta melhora de $50 \%$ do quadro de dor em $86 \%$ dos pacientes tratados.

\section{CONSIDERAÇÕES FINAIS}

A enervação tanto em humanos como em cães pode ser descrita como 
bilateralmente simétrica, não havendo diferença significativa entre seus antímeros. Os principais componentes da enervação humana são os ramos articulares dos nervos glúteo superior, isquiático, femoral e obturador, enquanto em cães, sua composição é baseada em feixes dos nervos glúteo cranial, isquiático e femoral.

A diferença do apoio bipedal plantígrado dos seres humanos e quadrupedal digitígrado dos cães sugere a diferença entre o número de ramificações nervosas e suas regiões, uma vez que nos animais a região mais enervada é aquela cuja responsabilidade é dos nervos glúteo cranial e isquiático, enquanto os seres humanos apresentam maior número de fibras sensitivas relativas ao nervo obturador.

\section{REFERÊNCIAS}

AKATOV, O.V.; DREVAL, O.N. Percutaneus radiofrequency destruction of the obturator nerve for treatment of pain caused by coxarthrosis. Stereotactic and Functional Neurosurgery. V. 69, n. 1-4. p. $278-80,1997$

BAUMGAERTNER, M.R. Fractures of the posterior wall of the acetabulum. The Journal of the American Academy of Orthopaedic Surgeons. V. 7. n. 1. p. 5465, 1999

BIRNBAUM,K.; PRESCHER, A.; HESSLER, S.; HELLER, K.D. The sensory innervation of the hip joint - an anatomycal study. Surgical and Radiological Anatomy. V. 19. n. 6. p. 371-375, 1997

FERRIGNO, C.R.A.; D'AVILA, R.S.; YAMAMOTO, E.; YASBEK,K.; FERRAZ, V.C.M. Estudo da técnica de denervação da cápsula articular no tratamento da dor em cães com displasia coxo-femoral: resultados preliminares. Brazilian Journal of Veterinary Research and Animal Science. V. 41 suppl. P. 169, 2004.

GARDNER, E.; The innervation of the hip joint. The Anatomical Record. V. 101, p. 353-371, 1948.

GOLUB, D.M.; BRONOVITSKAIA, G.M. Development of the Human hip joint and its innervation. Arkhiv Anatomii, gistologii I émbriologii. V. 80. n. 5. p. 47-56, 1981.

JACOBSON, A.; SCHRADER, S.C. Peripheral nerve injury associated with fracture or fracturedeslocation of the pelvis in dogs and cats: 34 cases. Journal of the American Veterinária Medical Association. V. 190. n. 1. p. 569-572, 1987

KAWAGUCHI, M.; HASHIZUME, K.; IWATA, T.; FURUYA, $H$. Percutaneous radiofrequency of sensory branches of the obturator and femoral nerves for the treatment of hip joint pain. Regional Anesthesia and Pain Medicine. v.26, n 6, p.57681.2001

KENNY, P.; O'BRIEN, C.P.; SYNNOTT, K.; WALSH, M.G. Damage to the superior gluteal nerve after two different approaches to the hip. Journal of Bone and Joint Surgery. V. 81. n. 6. p. 979-981, 1999.

KINZEL, S.; FASSELT, R.; PRESCHER, A.; SELZER, C.; v. KEYSERLINGK, D.G.; KÜPPER, W. Die sensible innervation der capsula articularis coxae beim hund. Tierarztliche Praxis. V, 26, p. 330335, 1998.

KINZEL, S.; HEIN, S.; v.SCHEVEN, C.; KÜPPER, W. 10 years experience with denervation of the hip joint capsule in the treatment of canine hip joint dysplasia and arthrosis. Berliner und Munchener Tierarztlich Wonchenschrift. V. 115, p. 53-56, 2002.

MACH, D.B.; ROGERS, D.; SABINO, M.C.; LUGER, N.M.; SCHWEI, M.J.; POMONIS, J.D.; KEYSER, C.P.; CLOHISY, D.R.; ADAMS, D.J.; O'LEARY, P.; MANTYH, P.W. Origins of skeletal pain: sensory and sympathetic innervation of the mouse femur. Neuroscience. V. 113, n.1, p, 155-166, 2002.

PUTZ, R.; SCHRANK, C.; Anatomy of the labrocapsular complex. Der Orthopäde. V. 27. n. 10. p. 675-680, 1998.

SCHMAEDECKE, A. Estudo quantitativo das fibras nervosas do periósteo acetabular em cães. 2004. 105 f. Dissetração (Mestrado) - Faculdade de Medicina Veterinária e Zootecnia, Universidade de São Paulo, São Paulo.

SCHMAEDECKE, A.; CASAS, A.L.S.; KOWALESKY, J.; ARTONI, L.P.; CARVALHO, R.C.; FERRIGNO, C.R.A. Estudo anatômico da enervação da articulação coxo-femoral em cães. In: XXX Congresso Brasileiro de Medicina Veterinária. Manaus. 2003. Anais

SIEBENROCK, K.A.;RÖSLER, K.M.; GONZALEZ, E.; GANZ, R. Intraoperative electromyography for the superior gluteal nerve during lateral approach to the hip for arthroplasty: a prospective study of 12 patients. The Journal of Arthroplasty. V.15, n.7, p. 867-870, 2000.

STASZYK, C.; GASSE, H. Zur innervation der gelenkkapseln beim hund. Teil 3 : hüftgelenk. Kleintierpraxis. V. 47, p 11-17, 2002. 